\title{
Entornos de Aprendizaje Eficaces con TIC
}

\author{
Efficient Learning Environments with ICT \\ Yira Muñoz-Sánchez ${ }^{a}$, María A. Alonso-Lavernia ${ }^{b}$, Iliana Castillo-Pérez ${ }^{c}$, Verónica Martínez- \\ Lazcano $^{d}$
}

\begin{abstract}
:
Learning environments have been conceived as a set of various elements that allow learning to be acquired. However, learning environments must meet certain characteristics such as allowing, at present, that the student assumes an active role and that through that environment they are offered the materials and tools that allow them to achieve their learning objectives, by what ICT and specifically Web 2.0 tools have come to favor this context to generate effective environments.
\end{abstract}

Keywords:

Learning Environments, ICT, Web 2.0, Efficient Learning Environments.

\section{Resumen:}

Los entornos de aprendizaje han sido concebidos como un conjunto de diversos elementos que permiten que se adquiera un aprendizaje. Sin embargo, los entornos de aprendizaje deben cumplir con ciertas características como permitir, en la actualidad, que el estudiante asuma un papel activo y que a través de dicho entorno se le ofrezcan los materiales y herramientas que le permitan lograr sus objetivos de aprendizaje, por lo que las TIC y específicamente las herramientas Web 2.0 han venido a favorecer este contexto para generar ambientes eficaces.

\section{Palabras Clave:}

Entornos de aprendizaje, TIC, Web 2.0, Entornos de Aprendizaje Eficaces.

\section{Introducción}

Gracias a el avance que han tenido las Tecnologías de la Información y Comunicación (TIC) en las últimas décadas, muchas áreas se han visto beneficiadas y sin duda, la educación ha sido una de ellas ya que, gracias al uso de nuevas herramientas tecnológicas, como las TIC, los entornos de aprendizaje han evolucionado para ser más eficaces.

\footnotetext{
a Autor de Correspondencia, Universidad Autónoma del Estado de Hidalgo, Escuela Superior Ciudad Sahagún, https://orcid.org/0000-00024876-2747, Email: yira@uaeh.edu.mx

b Universidad Autónoma del Estado de Hidalgo, Instituto de Ciencias Básicas e Ingeniería, https://orcid.org/0000-0002-9839-8250, Email: marial@uaeh.edu.mx

c Universidad Autónoma del Estado de Hidalgo, Instituto de Ciencias Básicas e Ingeniería, https://orcid.org/0000-0002-8130-9231, Email: ilianac@uaeh.edu.mx

${ }^{d}$ Universidad Autónoma del Estado de Hidalgo, Instituto de Ciencias Básicas e Ingeniería, https://orcid.org/0000-0003-2172-4000, Email: vlazcano@uaeh.edu.mx
} 


\section{Descripción}

Los entornos de aprendizaje combinan un conjunto de elementos que permiten generar el aprendizaje y para lograrlo deben incluir herramientas, fuentes de información confiables, el desarrollo de actividades y conexión para poder tener acceso a materiales actuales que se encuentran en internet. Para que un entorno de aprendizaje sea eficaz debe incluir sin duda, el uso de la TIC, ya que, gracias a los avances en las tecnologías, actualmente se cuenta con nuevos estándares, el acceso a cursos masivos y abiertos en línea, nuevos espacios y aulas de aprendizaje; regularmente son de tipo virtual o una combinación de lo presencial con lo virtual, software y multimedia y todo lo que incluye la tecnología web 2.0. El paradigma que coloca al alumno como centro del aprendizaje ha sido favorecido sin duda por las TIC, ya que, a través de las herramientas antes mencionadas, se puede favorecer el proceso de aprendizaje para que el alumno asuma un papel activo y la responsabilidad que le permita tomar las decisiones para el logro de los objetivos de aprendizaje.

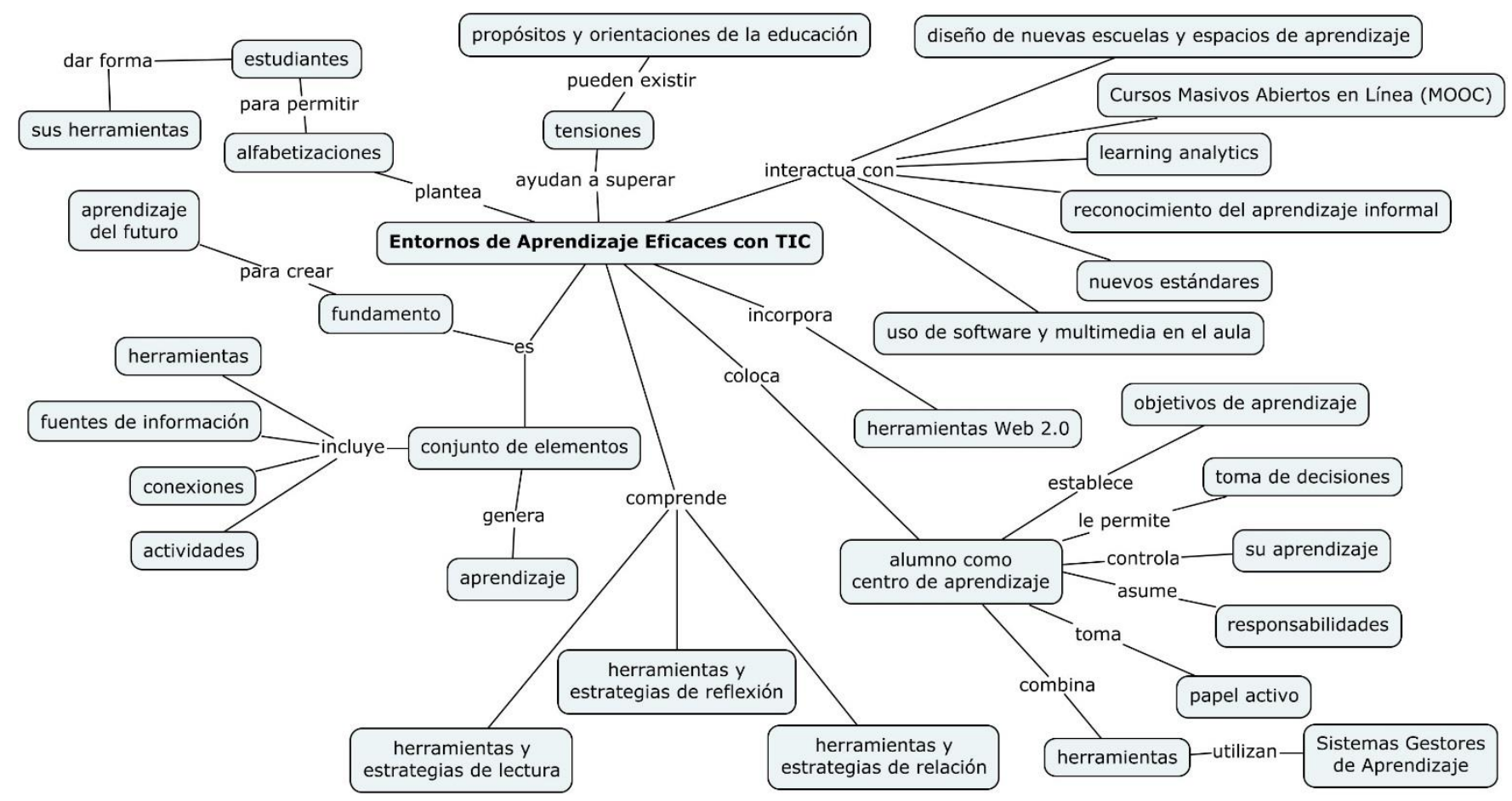

Figura 1. Entornos de Aprendizaje Eficaces con TIC.

\section{Referencias}

[1] Castañeda, L., \& Adell, J. (2013). Entornos Personales de Aprendizaje: claves para el ecosistema educativo en red. Alcoy: Marfil.

[2] Herrington, J., Oliver, R., \& Herrington, A. (2007). Authentic Learning on the Web: Guidelines. Flexible learning in an information society. In, 26-35.

[3] Herrington, A., \& Herrington, J. (2008). What is an authentic learning environment? Recuperado de https://goo.gl/984wAo.

[4] Pearlman, B. (2010). Designing new learning environments to support 21st century skills. 21st century skills: Rethinking how students learn, 116-147. Recuperado de https://goo.gl/fgfi8c

[5] Suárez, C. \& Serrano, J. (2016). Competencia digital y construcción de entornos personales de aprendizaje como retos de la formación universitaria. En C. Suárez-Guerrero; D. Marín-Suelves y D. Palomares-Montero (Coords.). Retos de la Educación en tiempos de cambio. Valencia: Tirant Humanidades (pp. 227-251). 